\title{
The first 10 years' experience of restorative proctocolectomy for ulcerative colitis
}

\author{
P Setti-Carraro, J K Ritchie, K H Wilkinson, R J Nicholls, P R Hawley
}

\begin{abstract}
Between 1976 and 1985, 110 patients had restorative proctocolectomy or proctectomy for ulcerative colitis and 103 were followed up until death or February 1992. There was one postoperative and one late death related to surgery. The cumulative probability of pouch failure was $12 \%$ at five years: half of the failures occurred within one year. The commonest reasons were perianal/pelvic sepsis and probable Crohn's disease. The cumulative probability of readmission, excluding that for ileostomy closure, was $68 \%$ at five years. There were 152 operations carried out during readmissions. These included 44 laparotomies. Function was assessed in 80 patients at a mean of 99.3 months after ileostomy closure. For 66 patients with spontaneous evacuation, average minimum diurnal frequency was $3 \cdot 8$, maximum 4.9, with 35 evacuating at night. One patient experienced major continence problems, 30 had minor leaks, and 49 were completely continent. Postoperatively, five patients gave birth to nine babies, four had renal stones, two myasthenia gravis, and two severe anaemia: seven had pre or postoperative thyroid dysfunction.

(Gut 1994; 35: 1070-1075)
\end{abstract}

The first restorative proctocolectomy in this hospital was carried out by Parks in 1976. Since that time it has become the most commonly used surgical procedure for ulcerative colitis as well as for selected patients with familial adenomatous polyposis and, more recently, some patients with functional bowel disease. With an experience of the operation of more than 15 years, it was felt appropriate to review the longterm outcome in patients with ulcerative colitis. To allow for lengthy follow up, the study has been confined to patients operated on before the end of 1985 . It was hoped that a minimum follow up of six years would permit an assessment of function in the long term and show any possible delayed adverse effects of the operation.

St Mark's Hospital, City Road, London P Setti-Carraro J K Ritchie $\mathrm{K} \mathrm{H}$ Wilkinson $R$ J Nicholls P R Hawley

Correspondence to: Mr R J Nicholls, St Mark' Hospital, City Road, Londo ECIV 2PS.

Accepted for publication 7 January 1994 Patients and methods patients were treated at this hospital for ulcerative colitis by restorative proctocolectomy or proctectomy. Review of the case notes was supplemented by personal examination of 66 patients by one clinician (PSC). Five patients were contacted by letter or telephone and for nine information was sought from the referring surgeon using a standardised proforma. Patients were followed up until death or February 1992, apart from seven $(6 \cdot 3 \%)$ who could not be traced at that time.

Fifty six patients had all their surgery performed at this hospital: 40 had a restorative proctocolectomy and 16 a restorative proctectomy. Fifty four patients were referred: 42 had been treated by a colectomy and ileostomy, four by a partial colectomy, and eight by colectomy and ileorectal anastomosis, which had proved unsuccessful (7) or in whom high grade dysplasia had developed in the rectum (1). A temporary ileostomy was constructed in 104 cases.

The indications for surgery in the 40 patients treated by a restorative proctocolectomy were chronic symptoms in 22 , severe relapsing disease in seven, dysplasia in eight, and known or suspected carcinoma in two. One patient (the first in the series) had the operation as an urgent procedure after failure of medical treatment for acute colitis.

Of the 16 patients undergoing a restorative proctectomy in this hospital, an original colectomy and ileostomy was carried out urgently in 10 and electively in four. In two patients, an ileorectal anastomosis was converted to a pouch for diarrhoea in one and high grade rectal dysplasia in the other.

There were 59 men and 51 women in the series with a mean age at operation of 31.6 years (median 30, range 14-60).

\section{TECHNIQUE}

Thirty five patients $(31.8 \%)$ had a three loop pouch (S), $34(30.9 \%)$ a two loop (J), 38 $(34.5 \%)$ a four loop (W), and three had a Kock reservoir without the inverted nipple valve. Mucosectomy of the anorectal stump was always performed and the ileoanal anastomosis was made endoanally to the dentate line.

\section{PATHOLOGY}

The pathological diagnosis was ulcerative colitis in 103 specimens and indeterminate colitis in three. In four patients there was insufficient rectum to permit a diagnosis to be made. Crohn's disease was not diagnosed in any specimen.

There were carcinomas in the specimen in seven patients: four had a single Dukes's A lesion, one a Dukes's B, and two had multiple tumours each with Dukes's C1 tumours as the most advanced. One patient had had two tumours (Dukes's A and B) in the colon before referral for restorative proctectomy. 
TABLE I Postoperative complications after restorative proctocolectomy or proctectomy (60 complications in 51 patients)

\begin{tabular}{ll}
\hline & Patients \\
\hline $\begin{array}{l}\text { No complications } \\
\text { Local complications }\end{array}$ & 59 \\
$\begin{array}{l}\text { (pelvic sepsis 8, minor anastomotic breakdown 6, } \\
\text { pouch-vaginal fistula 1, bleeding from drain 1, }\end{array}$ & 21 \\
$\begin{array}{l}\text { bleeding through anum 3, retraction of ileostomy 2) } \\
\text { General complications } \\
\quad \text { (small bowel obstruction 7 (laparotomy 2), chest } \\
\text { infection 2, deep venous thrombosis 2, septicaemia }\end{array}$ & 33 \\
$\begin{array}{l}\text { subphrenic abscess 1, myocardial infarction 1, } \\
\text { jaundice 2, raised ileostomy output 3, }\end{array}$ & \\
$\begin{array}{l}\text { wound infection 9, urinary tract infection 5) } \\
\text { Other complications } \\
\text { Death (bilateral pulmonary collapse and effusions) }\end{array}$ & 1 \\
\hline
\end{tabular}

\section{POSTOPERATIVE COMPLICATIONS}

There was one postoperative death: the patient died on the evening of the operation from bilateral pulmonary collapse with massive pleural effusions. Fifty nine patients had no complications. Table I shows the complications in the remaining 50 patients. Significant pelvic sepsis occurred in eight $(7 \cdot 3 \%)$ patients: in no case was laparotomy required but in four a pelvic abscess needed drainage through the anum under anaesthetic. The mean postoperative stay after the ileoanal procedure was 17 days (median 14, range 7-65).

\section{CLOSURE OF THE ILEOSTOMY}

The ileostomy was closed in 101 of 104 patients and the mean interval between this and the ileoanal procedure was 96 days (median 71, range 21-399). Nine patients developed small bowel obstruction and four required laparotomy. Wound infection occurred in nine patients, one patient had upper gastrointestinal bleeding (coffee ground aspirate from nasogastric tube), and one developed an ischiorectal abscess. Overall there were 20 complications in 19 patients. The mean postoperative stay was 14 days (median 11, range 4-67).

The ileostomy was not closed in three patients. These were the patient who died postoperatively, a patient in whom a rectal carcinoma was found in the specimen after restorative proctectomy and the pouch

TABLE II Failure of restorative proctocolectomy or proctectomy among 109 operation survivors

\begin{tabular}{llll}
\hline $\begin{array}{l}\text { Patient } \\
n o\end{array}$ & Reasons for failure & $\begin{array}{l}\text { Interval (months) } \\
\text { from ileostomy } \\
\text { closure }\end{array}$ & \\
\hline $1^{\star}$ & Recurrent pelvic and perianal sepsis ?CD & - & Treatment \\
$2^{\star}$ & Rectal cancer, inadequate clearance of margins & - & Excised \\
3 & Pouch-vaginal fistula & 0 & Excised \\
4 & Recurrent pelvic sepsis & 0 & EI \\
5 & Incontinence, frequency, pouchitis & 1 & Excised \\
6 & Perianal sepsis ?CD & 5 & Excised \\
7 & Pouch defect, small bowel, and anal fistulas ?CD & 6 & Excised \\
8 & Incontinence, perianal sepsis & 11 & Excised \\
9 & Recurrent pelvic and perianal fistula ?CD & 12 & Excised \\
10 & Perianal sepsis, probable pouch-vaginal fistula ?CD & 13 & Excised \\
11 & Malaise, frequency & 25 & Excised \\
12 & Malaise, failure to thrive & 33 & Excised \\
13 & Recurrent pouchitis, frequency & 36 & EI \\
14 & Recurrent pelvic sepsis and anal fistula ?CD & 46 & KI \\
15 & Incontinence, frequency & 80 & KI \\
16 & Incontinence, frequency & 83 & EI
\end{tabular}

$\mathrm{CD}=$ Crohn's disease, $\mathrm{EI}=$ end ileostomy, $\mathrm{KI}=$ Kock ileostomy, * = ileostomy not closed before pouch excision, ${ }^{\star \star}=$ Kock ileostomy subsequently excised. removed two weeks later (case no 2, Table II), and a patient in whom the pouch was ultimately removed (case no 1 , Table II).

The mean duration of hospital stay for both procedures combined was 30 days (median 26 , range $13-81$ ).

\section{MORTALITY}

In addition to the one postoperative death, there was a further death related to surgery. This patient died of septicaemia 17 months after restorative proctectomy. Four patients died of carcinoma at intervals between 24 and 61 months after surgery. Three patients died of unrelated causes (road traffic accident, carcinoma of the stomach, and grand mal seizures) at intervals of 30,89 , and 151 months respectively from the operation.

\section{FAILURE}

Failure was defined either as the need to remove the reservoir, to defunction by an ileostomy not closed at the time of this assessment or to convert to a Kock abdominal reservoir. There were 16 such cases giving an overall crude failure rate of $15 \%$ (Table II). The cumulative probability of failure from the time of ileostomy closure (based on the last 14 patients in Table II) was $12 \%(95 \%$ CI $7 \%$ to $20 \%$ ) at five years and $15 \%(95 \%$ CI $9 \%$ to $24 \%$ ) at seven years (Figure). Among the patients in whom the pouch failed, Crohn's disease was suspected in seven: in two biopsy of an anal lesion supported this diagnosis. Perianal pelvic sepsis, or both, were the commonest reasons for failure. The result was satisfactory in one of the three patients converted to a Kock continent ileostomy: in the other two the abdominal reservoir was excised. There was no statistically significant difference in the failure rate in the two periods 1976-80 (4 of 19: 21\%) and 1981-85 (12 of $90: 13 \%), \mathrm{p}=0 \cdot 47$.

\section{Readmissions}

During the period of follow up before or after ileostomy closure $74(68 \%)$ patients were readmitted on 188 occasions here or elsewhere excluding the admission for closure of the ileostomy. These figures are up to February 1992 or up to and including the admission at which the pouch failed. Thirty three patients were readmitted once, 15 twice, and 26 on three or more occasions. Surgical treatment was done during 127 of these readmissions. The operations were abdominal in 63 (including 44 laparotomies) and confined to the pouch or anal region in 89 . The risk of readmission from the time of formation of the pouch was $68 \%$ at five years ( $95 \%$ CI $59 \%$ to $77 \%$ ).

\section{POUCHITIS}

Pouchitis was diagnosed by the presence of frequency of defecation associated with endoscopic appearances of inflammation and histological evidence of inflammation in a 


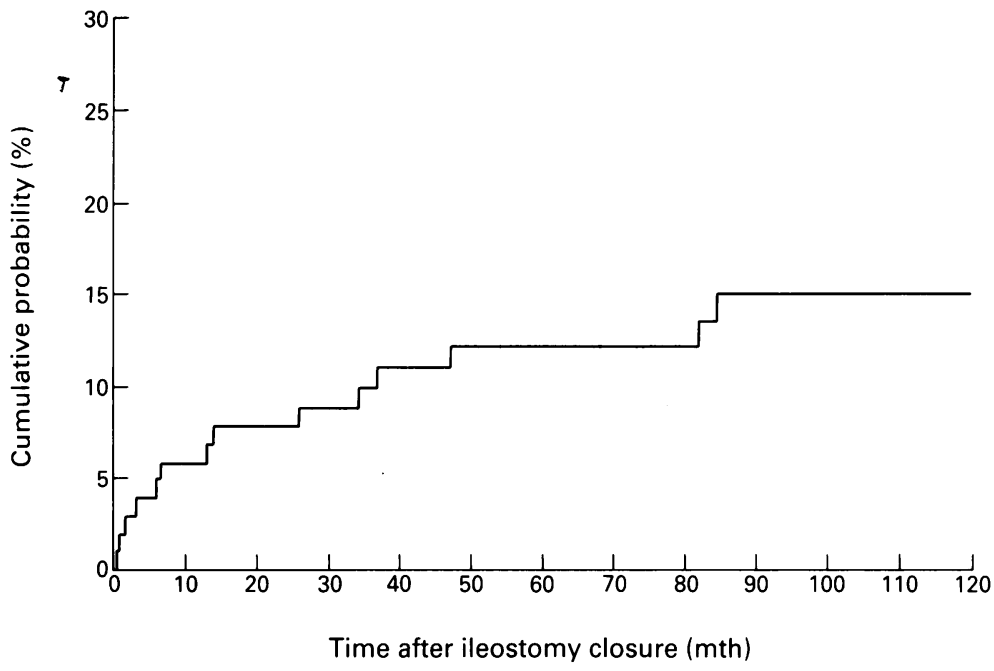

Cumulative probability of pouch failure after ileostomy closure.

biopsy specimen from the reservoir. Complete information was available for 60 of 66 patients seen in the clinic. Of these, 30 had never had any evidence of pouchitis, 21 patients had had at least one attack, and nine had chronic persistent pouchitis.

\section{FUNCTION}

At the time of the study there were 80 patients with an intact pouch available for a review of function. The mean follow up from closure of the ileostomy was 99.3 months (range 63-173). There were 24 patients with, an $S$ pouch, 24 with a J, 31 with a $W$, and one with a pelvic Kock pouch.

Sixty six patients did not use a catheter. Average diurnal minimum frequency among these was 3.8 (range 1-10) and maximum of 4.9 (range 1-12).

Of the 66 patients with spontaneous evacuation, $31(47 \%)$ never had a bowel action at night. In 21 , bowel movements occurred sometimes during the night and in 14 every night. Of the 35 patients, 24 had at most one bowel action, eight had two, two had three, and one patient had four.

Overall, the average minimum 24 hour frequency in these 66 patients was 4.1 and the maximum $5 \cdot 7$.

Forty nine patients were completely continent but one wore a pad as a precaution. Of the remaining 31,30 had minor mucous or faecal leaks. One patient had more severe faecal leaks, particularly at night. Of these patients, 23 needed to wear a pad. Twenty seven patients took constipating agents.

Urgency was a rare complaint: of the six patients who could not defer evacuation for 15 minutes, three had active pouchitis at the time.

Fourteen of 24 patients with an $S$ pouch needed to use a catheter. None of the other 56 patients with an $S$ or other type of pouch needed to use a catheter. impairment of sexual function. In one man of
30 years this was temporary and had resolved completely a year after closure of the ileostomy. In the other patient, aged 60 years at the time of restorative proctocolectomy for a rectal carcinoma complicating ulcerative colitis, lack of complete erection was thought to result from Peyronie's disease and was treated by a penile implant.

Five of 37 women became pregnant after the operation and gave birth to nine babies: all but two deliveries (in the same patient) were by caesarean section.

GENERAL HEALTH

Longterm morbidity in these patients seemed to fall into four categories, two probably related to the surgery and two apparently unrelated.

Anaemia with an iron deficiency picture was sufficiently severe in three patients to require transfusion; in two there had been significant blood loss as a result of pouchitis.

Renal calculi were recorded in four patients. One patient passed a stone about six weeks after restorative proctocolectomy and another patient had a left renal calculus some seven years after a restorative proctectomy. One patient was found to have a $2 \mathrm{~cm}$ calculus in the left renal pelvis while an inpatient after excision of the pouch and was treated medically with a diagnosis of hyperuricaemia. The fourth patient had passed a stone two years before restorative proctectomy and six years after the pouch operation, a large right sided renal calculus was treated by lithotripsy.

Seven patients had various thyroid disorders, three before the pouch operation. One was said to have been hypothyroid in the past, one had had a thyroidectomy and was receiving thyroxine, and one had acute thyrotoxicosis treated medically and by radioactive iodine and five years after the pouch was receiving thryoxine for hypothyroidism. At the time of restorative proctectomy, one patient was found to have a symptomless bilateral multinodular goitre and another had a nodule in the left lobe of the thyroid with normal thyroid function tests.

Two patients developed thyroid disorders after the restorative procedure, one hyper and one hypothyroidism at intervals of six years and six months respectively. Both had other diseases: the first renal sarcoid some three months after a restorative proctocolectomy having had pulmonary involvement eight years earlier. The second who had had two colonic carcinomas at the original colectomy elsewhere, developed the first signs of myasthenia gravis while an inpatient at this hospital after the pouch operation. Thymectomy was carried out six months later.

One other patient also developed myasthenia gravis just over three years after restorative proctocolectomy. Treatment with azathioprine and prednisolone was continuing four years later when he was last seen. 
LIFE EXPECTANCY

Excluding all patients with carcinoma, the five year survival probability after the restorative operation was $98 \%(95 \%$ CI 90.5 to $99 \%)$ and the 10 year figure was $95.5 \%$ (95\% CI 88.3 to $98 \cdot 3 \%$ ).

The number of observed deaths was four (one related late death resulting from sepsis and three resulting from unrelated causes) or five if the postoperative death is included. The number of expected deaths was 1.09 giving a ratio of 3.7 for observed to expected deaths with $95 \%$ CI of 1.0 to $9 \cdot 4, p=0.05$.

\section{Discussion}

Any elective operation for ulcerative colitis should be associated with a nil or very low postoperative mortality, the initial and longterm complication rate should be acceptable, and the disease eradicated in total or near total with the carcinoma risk eliminated or very much reduced. Restorative proctocolectomy retaining the normal faecal pathway comes nearest to the ideal and has the advantage over colectomy and ileorectal anastomosis in that it can be used in patients with severe rectal disease. Over the past 15 to 20 years at this hospital, it has become the most common operation in the elective situation or, as a two stage procedure, for patients operated on urgently. ${ }^{1}$

Restorative proctocolectomy at this hospital is generally restricted to younger patients. The mean (variance) age at operation of the first 110 patients treated here between 1976 and 1985 is identical to that of 390 cases from the Mayo clinic operated on from 1981-85 (31 (9 years)), ${ }^{2} 82$ from Toronto, ${ }^{3}$ and 114 from Minneapolis ${ }^{4}$ although the last two series contained patients with familial adenomatous polyposis. The average age at operation of patients treated by excisional surgery at this hospital was $40 \cdot 7$ years $^{5}$ but, since the advent of restorative proctocolectomy, has risen to 49 years for elective total proctocolectomy. ${ }^{6}$

All operations for ulcerative colitis are major undertakings even when elective and the one postoperative death $(0.9 \%)$ in this series compares with one such death in 390 patients $(0.3 \%)$ in the largest single series ${ }^{2}$ although there has been no such mortality in many others. ${ }^{37-9}$ Both elective total proctocolectomy and colectomy and ileorectal anastomosis also carry a postoperative mortality. ${ }^{110}$

Postoperative complications can be divided into those of any operation and those relating to the specific procedure. In this series, the complication rate was high: 51 of 110 patients had complications. Complication rates from other series range from $13 \%{ }^{7}$ upwards with the largest series having a combined postoperative complication rate from the initial operation and ileostomy closure of $29 \% .^{2}$ The general complications were those associated with any abdominal operation with the most frequent (apart from wound infection) being small bowel obstruction in seven patients requiring laparotomy in two. This obstruction rate of $6 \%$ and needing surgery in $2 \%$ is low in comparison with some reports. ${ }^{2} 8$ Of the local complications pelvic sepsis in eight patients $(7 \%)$ was the most frequent with four needing drainage of an abscess, but without laparotomy. The figure of $7 \%$ falls well within the range reported from elsewhere. ${ }^{2811}$ The overall complication rate of $45 \%$ is not dissimilar to that of $39 \%$ for elective total proctocolectomy over almost exactly the same time period at this hospital. ${ }^{6}$ Furthermore the median postoperative stay for the restorative operation was 14 days compared with 16 for the excisional surgery: patients in the second group were, however, considerably older.

This is, of course, a misleading comparison, as, in most patients, a second operation was required for closure of a temporary ileostomy with a median postoperative stay of 11 days and a complication rate of $19 \%$. The small bowel obstruction rate after this second operation was $9 \%$ with a reoperation rate of $4 \%$ : these figures are almost identical with those from elsewhere. ${ }^{2}$

Reported series do not give detailed figures for readmissions during the follow up period and, as far as we are aware, there had been no calculation of the cumulative probability of readmission previously. In addition, the mean duration of follow up in the largest series ${ }^{2}$ was only 2.3 years compared with 6.6 years in this report. Thus the probability of readmission of $68 \%$ at five years, which seems high cannot be compared with that from elsewhere. Perhaps the fairest comparison is with that for elective total proctocolectomy at this hospital over a very similar time period, which was $45 \%$ at six years. ${ }^{6}$ The longterm course of patients after restorative proctocolectomy should be viewed against that for total proctocolectomy and never in isolation.

The overall failure rate of the operation (including three patients with a disconnected pouch) was $15 \%$, which was disappointing but perhaps not entirely surprising as we are reporting here the first 110 patients with ulcerative colitis in an experience at this hospital that now encompasses over 400 cases. Failure rates in other series range from $0-14 \%$ : an overall rate in nine series was $5 \% .{ }^{12}$ These, however, were mainly early and the failure rate clearly increases with the duration of follow up. The reasons for early failure in our patients were similar to those reported elsewhere - that is, pelvic sepsis, incontinence, intractable pouchitis, and possible Crohn's disease. The last, suspected in seven of our cases, was never diagnosed in any specimen and only the subsequent clinical course suggested the diagnosis. Most of the failures occurred fairly soon after ileostomy closure as reported by others. ${ }^{2911}$ In the long term, however, failures occurred for various reasons including poor function, malaise, and fistulation from the reservoir. Pouch-vaginal or perineal fistula is often delayed several months after ileostomy closure and accounts for some late failures. ${ }^{13}$ These have not been included in most reports of the outcome of restorative proctocolectomy.

In this series, postoperative obstruction 
requiring surgery was similar to that after excisional surgery but the rate $(18 \%)$ during follow up may be higher. Now that small bowel obstruction is a well recognised complication of all operations for ulcerative colitis both in the short and the long term, it is to be hoped that it will no longer be a cause of late death as previously. 1014

Anastomotic stenosis has become a well known long term complication in pouch patients. Seven patients in this series (with 14 admissions) required dilation under general anaesthetic for this complication. Other reports give similar incidences ranging from $5-18 \%$ with an overall incidence with nine series of $14 \cdot 7 \% .^{211} 12$ Stenosis, however, was not a factor leading to pouch excision in our patients as in the Birmingham report. ${ }^{11}$

At this hospital, pouchitis has been diagnosed by clinical, endoscopic, and pathological criteria. Frequency of defecation alone is insufficient to justify the diagnosis but must be accompanied by the presence of acute inflammation on biopsy examination. ${ }^{15}$ Only 60 of our patients were thus fully assessed and, of these, half had never had evidence of pouchitis. Of the 21 with one episode, this had resolved with treatment (usually metronidazole) and had not recurred. Nine patients with persistent pouchitis had a variety of treatments including low dose longterm metronidazole and conventional anti-inflammatory treatment. In only two $(12.5 \%)$ of 16 patients who failed was intractable pouchitis the major reason.

The details of function reported here relate to patients after at least five years from closure of the ileostomy. It is known that function can improve for several years after operation and so our figures must represent the optimum that has been achieved. Comparison with other series may not be valid because of different lengths of follow up and varying pouch design. Our results include four types of pouch and overall figures are given. A minimum diurnal stool frequency of 3.6 (range 1-10) and a maximum of $4 \cdot 7$ (range of 1-12), however, compares well with other series, for example, 6 (3) at three years in the Mayo series, ${ }^{2} 5(1.5)$ at six months, ${ }^{3}$ and $5.4(2.5)$ at mean follow up of five years. ${ }^{4}$ Almost half of the patients $(47 \%)$ in this series never had a bowel action at night: this compares with a figure of $73 \%$ from elsewhere. ${ }^{3}$ Other reports do not give a comparable figure.

Minor mucous or faecal leakage occurred in some $40 \%$ of patients but only a third of these (around $16 \%$ of the total) needed to wear a pad. This is in noticeable contrast with another series where $62 \%$ usually used a pad by day and $68 \%$ at night. ${ }^{4}$

Perhaps the most important aspects of this follow up are the enquiry into general health and the life expectancy figures, which have not been mentioned previously.

Minor degrees of anaemia are sometimes seen in patients with pouches and the picture is usually one of iron deficiency that can be corrected with oral iron. A low serum B-12 resulting possibly from changes in the terminal ileum because of the pouch may also need appropriate treatment. Severe degrees of anaemia with haemoglobin as low as $5.0 \mathrm{~g} / \mathrm{dl}$ in two of our patients are more worrying particularly as it was recurrent in one patient and the cause never adequately established.

A tendency to urolithiasis, perhaps greater than that of the general population has been shown in patients after total proctocolectomy 5 16 and the occurrence of stones in four patients may relate to the previous ileostomy in two and a high output from a defunctioning ileostomy in one.

Although seven patients had thyroid disorders before or after the establishment of the pouch, these were of various types and probably unrelated to the operation. Thyrotoxicosis in a patient with ulcerative colitis has been described $^{17}$ and there seems no reason why these two disorders should not, on occasion, occur in the same patient.

Two cases of myasthenia gravis among 109 patients who left hospital after the pouch does, however, seem strange as myasthenia is an uncommon disease. No conclusions can be drawn from two cases: a longer follow up may clarify the position.

With certain provisos, it is known that life expectancy is normal in patients after excisional surgery for colitis. ${ }^{5}$ For colectomy and ileorectal anastomosis the number of observed deaths was over twice the expected with 12 deaths resulting from rectal carcinoma. ${ }^{10}$ With strict follow up and routine use of rectal biopsies, it is to be hoped that better figures could be obtained nowadays.

The findings after restorative proctocolectomy are encouraging. Patients with carcinoma in the specimen were excluded and thus the only related late death was from sepsis. The figure of 3.7 of observed/expected death lies within the $95 \%$ confidence intervals. This suggests that the operation is compatible with a normal life expectancy in the short term but much longer follow up is necessary before this can be said with certainty for the long term.

1 Melville DM, Ritchie JK, Nicholls RJ, Hawley PR. Surgery for ulcerative colitis in the era of the pouch: the St Mark's Hospital experience. Gut 1994; 35: 1076-80.

2 Pemberton JH, Kelly KA, Beart RW, Dozois RR, Wolff BG, Ilstrup DM. Ileal pouch-anal anastomosis for chronic ulcerative colitis. Ann Surg 1987; 206: 504-12.

3 Cohen Z, McLeod RS, Stern H, Grant D, Nordgren S. The pelvic pouch and ileoanal anastomosis procedure. Surgical technique and initial results. Am f Surg 1985; 150: $601-7$.

4 Wexner SD, Jensen L. Rothenberger DA, Wong WD, Goldberg SM. Long-term functional analysis of the ileoanal reservoir. Dis Colon Rectum 1989; 32: 275-81.

Ritchie JK. Ulcerative colitis treated by ileostomy and excisional surgery. Fifteen years' experience at St Mark's hos-
pital. Br f Surg 1972; 59: 345-51.

Phillips RKS, Ritchie JK, Hawley PR. Proctocolectomy and ileostomy for ulcerative colitis: the longer term story. $\mathcal{F} R$ Soc Med 1989; 82: 386-7.

7 Becker JM, Raymond JL. Ileal pouch-anal anastomosis. A single surgeon's experience with 100 consecutive cases. Ann Surg 1986; 204: 375-81.

8 Pescatori M, Mattana C, Castagneto M. Clinical and functional results after restorative proctocolectomy. Br $\mathcal{F}$ Surg 1988; 75: 321-4.

9 Wexner SD, Wong WD, Rothenberger DA, Goldberg SM. The ileoanal reservoir. Am $\mathcal{F}$ Surg 1990; 159: 178-83.

10 Baker WNW, Glass RE, Ritchie JK, Aylett SO. Cancer of the rectum following colectomy and ileorectal anastomosis for ulcerative colitis. Br f Surg 1978; 65: 862-8.

11 Keighley MRB, Winslet MC, Flinn R, Kmiot W. 
Multivariate analysis of factors influencing the results of

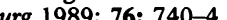
restorative proctocolectomy. Br $\mathcal{f}$ Surg 1989; 76: 740-4.
Williams NS, Johnston D. The current status of mucosal proctectomy and ileo-anal anastomosis in the surgical treatment of ulcerative colitis and adenomatous polyposis. Br $\mathcal{F}$ Surg 1985; 72: 159-68.

13 Groom JS, Nicholls RJ, Hawley PR, Phillips RKS. Pouchvaginal fistula. Br $\mathcal{F}$ Surg 1993; 80: 936-40.

14 Ritchie JK. The cause of late mortality in ileostomists. Proc $R$ Soc Med 1972; 65: 73.
15 Madden MV, Farthing MJG, Nicholls RJ. Inflammation in ileal reservoirs: 'pouchitis'. Gut 1990; 31: 247-9.

16 Ritchie JK. Ileostomy and excisional surgery for chronic inflammatory disease of the colon: a survey of one hospital region. Part II The health of ileostomists. Gut 1971; 12: $536-40$.

17 Triantafillidis $\mathrm{JK}$, Manoussakis $\mathrm{CA}$, Tsafaras $\mathrm{Ch}$, Koutsorizof A. Coexistence of thyrotoxicosis and exacerbation of ulcerative colitis. Am f Gastroenterol 1990; 85: 908-10. 